\title{
A qualitative study of mealtime difficulties in patients with dementia who feed themselves
}

\author{
DGH Damayanthi, AMNL de Silva, IAGMP Gunathilake, CU Suraweera
}

\section{Abstract \\ Background}

Caregivers looking after persons with dementia may often experience mealtime difficulties. This has been described in persons with severe dementia in the West. But data from Asian countries such as Sri Lanka is limited, and there has been less focus on problems associated with the care of people with mild or moderate dementia.

Aims

The aims of this study were to describe mealtime difficulties experienced by caregivers of individuals with dementia who feed themselves, and the responses by the caregivers to these problems.

\section{Methods}

A focus group discussion was conducted with the participation of twelve caregivers of individuals living with dementia who feed themselves and are receiving home based care. A pretested semistructured discussion guide was used. An abridged transcript was developed from the audio tape recording, transcribed notes and observations by the moderator. Braun and Clarke's six step approach to thematic analysis was adopted in the analysis.

\section{Results}

The main themes identified were; problems experienced by the caregivers when preparing food, changes in quantity of food consumed, measures taken by caregivers to overcome mealtime difficulties and the emotional responses of the caregivers. Mealtime difficulties can lead to reduced intake as well as overeating. Voluntary overfeeding by the caregivers was observed. Modification of dining environment or use of adaptive utensils were not practiced. Emotional exhaustion among the caregivers, and humiliating or dishonouring the individuals with dementia were the maladaptive responses.

\section{Conclusions}

Some of the mealtime difficulties in individuals with dementia in the Sri Lankan setting differ from those described in international literature. Early recognition of difficulties and guidance on adaptive measures will enable independence for a longer period and better nutrition.

Key words: dementia, mealtime, nutrition, feeding

SL J Psychiatry 2020; 11(1): 26-31

\section{Introduction}

Dementia is characterised by progressive deterioration of one or more cognitive domains, and the individual ultimately often loses independence in his or her daily activities (1). Dementia is associated with physical, psychosocial and financial implications to the caregiver, family and society $(2,3)$.

A range of mealtime difficulties can be observed in people with dementia and this varies according to the stage of the illness $(4,5)$. Under-nutrition and weight loss are common among people with dementia (6). Many publications have addressed these issues among individuals with advanced dementia (7-9). Mealtime difficulties encountered by individuals who are still feeding themselves and receiving home-based care have been overlooked. Available information on issues related to mealtime and remedies in early disease is restricted to Western societies. Mealtime difficulties vary remarkably in Asian societies due to differences in setting of care, cultural differences and food selection. In the Asian setting, most patients receive home based care, and family members are often caregivers (10). Therefore, recognition of common problems in this setting would enable clinicians to address these better in their routine clinical practice. 
The objectives of this focus group discussion were to identify mealtime difficulties experienced by caregivers of individuals with dementia who are feeding themselves, and to identify the responses of caregivers to these problems.

\section{Methods}

This was a qualitative study, using a focus group discussion. Prior to conducting the discussion, a semistructured discussion guide was formulated based on the existing literature and the study objectives. The discussion guide was then translated to Sinhala and pretested on two separate caregivers of individuals living with dementia. The key discussion topics and questions were as follows:

- What happens during mealtime and what kind of support is required?

- How do mealtime problems progress with time?

- What kind of support do you think is needed at each stage?

- Have you used any special utensils to assist the patient to continue feeding themselves?

- Have you ever experienced any emergency situations during mealtimes?

- What are the sources of support you use to overcome mealtime issues?

Participants were recruited from the University Psychiatry Clinic of National Hospital of Sri Lanka. During a training program conducted for caregivers, conducted at the above clinic, the reasons and objectives of the study was clearly described, and an open invitation was given to all caregivers of individuals with dementia who were feeding themselves. Twelve regular caregivers who gave informed consent were recruited to the study. All were fluent in the Sinhala language.

The focus group discussion was carried out in August 2018 , in a quiet venue with few external distractions. The discussion was facilitated by a moderator who was trained in clinical nutrition. Anote taker (trained in geriatric psychiatry) took the notes on participants' mood, and nonverbal cues. The discussion was audio taped. The discussion was carried out in Sinhala, for a duration of 55 minutes.

\section{Analysis}

An abridged transcript was formulated immediately following the discussion. Audio taped recordings, notes of the note taker and observations of the moderator were used to formulate the transcript. Braun and Clarke's six step approach to thematic analysis was adopted in analysis of transcript (11). Analysis was started after familiarization with the data by reading and re-reading the whole transcript. Coding of the data was done with colour pens. After two investigators reviewed the preliminary themes, and codes under each theme, the main themes and sub themes were identified. When writing the results, selected phrases were translated in to English by an investigator and retranslated into Sinhala by a separate investigator to assess comparability with original content.

\section{Ethics}

Ethics approval for the study was obtained from Ethics Review Committee, of the National Hospital of Sri Lanka. Only those who gave written informed consent were included in the study. All audio and hardcopy data was stored securely and confidentially.

\section{Results}

\section{Participant characteristics}

Caregivers of persons with mild to moderate dementia were included in the study. All the caregivers were family members and all had been with the person from the diagnosis of the illness (Table 1).

Table 1. Details of the caregivers included in the
study, and the persons with dementia being
looked after by the caregivers

Out of 12 individuals with dementia being cared-for, one was able to prepare food with the assistance of the caregiver. None of the others were preparing meals and they needed varying levels of assistance at mealtime, ranging from minimal support to continuous supervision and encouragement.

Four main themes and sub themes were recognised. These themes are interrelated and not mutually exclusive. 
Problems experienced by the caregivers with regards to preparation of food and at mealtimes

Difficulty in food preparation was one area of concern. Some of the caregivers were caring for spouses with dementia, and faced problems in having to make regular meals. One of the caregivers who was looking after his wife described it this way:

"She was the one who used to cook for both of us. Now she can't cook alone, so I help her to cook".

A majority of the individuals described in the study did not have any problem in getting their food prepared, as the caregiver or the other family members were preparing food.

We noticed that difficulties in continuing mealtime habits was another concern. Some were not used to sit at the table to dine. They sat on a chair in front of the television and took meals while holding the plate with one hand, which became difficult with time. One participant of the group discussion described it this way:

"It was difficult for him to sit on the chair while holding the plate with his hand, the plate tilts to a side and food falls on the floor. Therefore, I make him sit first and give the plate to his hand. Yet he cannot eat while holding the plate with one hand anymore......"

Maintaining table manners has become difficult for many persons with dementia, and this was a concern for the caregivers. We came across such a situation where caregiver described that her husband was not following table manners.

"........he takes spoon with the hand he was eating with”.

\section{Changes in the quantity of food consumed}

One common theme that emerged was regarding changes in the quantity of food consumed. Some carers noted decreases in food intake. Here the carer describes how the patient's passivity and need of assistance led to reduced intake:

"When he is made to sit at the table he stays still. He doesn't serve himself....... When he is eating he misses the curries and leaves them on the plate".

Increased intake of food was also described by some carers. Reduced awareness of the quantity of food led to over eating, if not actively prevented by the caregiver. One of the caregivers described that her mother had no idea of the quantity of food to serve, when eating:

"If I allow her to serve, she takes all the food from the pot".
Another caregiver described how her mother would finish a whole bunch of bananas if it was kept in an accessible place:

"If the bananas are kept on the table she finishes all of it".

When the food and beverages were served to plates or cups for the person with dementia as well as other family members, the person with dementia would consume the others' food and beverages due to the difficulty in recognizing own utensils. One of the caregivers described how this was a problem:

"He cannot identify his cup, he drinks the tea made for children if I keep those in a place where he can see".

As the person with dementia often could not recall whether they have had meals or not, offering food more than once in a single meal results in overeating. One caregiver did not identify this as a problem:

"He cannot remember whether he ate or not, if I ask he says: no I didn't eat.... So I don't mind giving food again".

\section{Measures taken by the caregivers}

Some caregivers planned meals to reduce the workload and stress, and to ensure regular meal patterns. One caregiver who was living alone with his spouse elaborated on how they were managing this situation:

"... I help her to cook. We cook for a whole week and store in the fridge".

Changing the dining to a more convenient place was another adaptive measure described in the group. The caregiver who described that her husband used to sit in front of television to dine while holding the plate, further elaborated as below:

"I used to make him sit first and give the plate to his hand. Yet he cannot eat while holding the plate with one hand anymore. Now I offer him his plate at the table".

Individuals who were passive at the table and were unable to complete the meal alone appeared to benefit by supervision and assistance. One of the caregivers whose husband tended to stay passively at the table described how she assisted him:

"....When he is eating he misses the curries and leaves them on the plate. I have to point them out to him".

Another caregiver described how she used to keep other family members' cups separately to avoid confusion. 
Adaptive utensils enable individuals to be independent to a certain degree. One caregiver had introduced a metal plate and a cup in the place of fragile porcelain utensils, to avoid breakage.

"Now I give food in a metal plate and cup".

In this study population no one was using cutlery.

While many of the measures taken by the caregivers were adaptive, we noticed that some caregivers have adopted non-adaptive measures. Some of the caregivers reported taking over responsibilities as a response to functional deficits and mistakes, instead of helping the individual to be independent. For example, two of the caregivers described that they now served food for their relative with dementia:

"I serve food to her plate. If I allow her to serve she takes all the food from the pots".

"It is difficult for her to serve-so I do it for her".

\section{Emotional responses of the caregivers}

Exhaustion was very well apparent in one of the caregivers. She lived alone with her husband with dementia. She expressed being exhausted with carrying out all the household work and looking after her husband.

"How can I do these things all alone? I am also a patient. I have lost my weight too”.

In response to mistakes, some individuals with dementia would be criticized by the caregivers. For example one caregiver criticized her husband's inability to maintain table manners:

"How much I tell he takes the spoon with the hand he was using to eat his food".

Some were humiliated because of the difficulties they face at mealtimes. One of the caregivers stated that she found it funny that her husband was drinking tea made for others.

One caregiver reported that she gave her husband food on a metal plate and a cup instead of porcelain plate. Even though she had done it as an adaptive measure it was apparent that she felt he was dishonoured by this action. This emotional response was revealed to us from her tone and use of words.

"Now I give food in metal plate and cup. Previously he didn't eat in those, he used porcelain ...."

Caregivers denied experiencing emergency situation at mealtimes.

None of the participants had sought professional assistance from support groups for mealtime difficulties. Caregivers had not considered these difficulties as a clinical problem.

\section{Discussion}

Our focus group discussion provides insight into mealtime difficulties experienced by caregivers of people with dementia and their responses, from a South Asian perspective. This study addresses difficulties among people with dementia who are able to feed themselves, whereas the available literature focuses mostly on people with advanced dementia.

The main themes that emerged from the study included, difficulties in continuing mealtime habits and manners, difficulties in preparing food and changes in quantity of food intake leading to reduced intake as well as overeating. Some caregivers took over responsibilities, making the individual with dementia more dependent. A range of emotional responses on the part of the caregivers was also observed.

Weight loss in people with dementia is well documented (12). Factors which can influence the quantity of food intake in those with advanced dementia have been identified, such as chewing difficulties, swallowing difficulties and refusal to feed. We did not come across these issues, probably because the group we selected only included people with early dementia $(13,14)$.

The literature documents that people with dementia ingest more calories per kilogram body weight within a day compared to people of the same age without dementia (15). We identified several possible reasons for overeating in the current study. Impaired judgment on food quantity and difficulty in recognizing their own utensils are possible causes. Voluntary overfeeding by caregivers noted in our study is not reported in the literature but may also be contributing to overfeeding. This behaviour on the part of the caregivers may be due to the cultural view that providing as much as food as possible to an 'ill' individual is considered an act of merit.

Mealtime practices vary among cultures, which influences the difficulties in feeding themselves, experienced by patients with dementia. For example serving food directly from the cooking pots to the plate and sitting on a chair to dine while holding the plate with one hand, are recognized practices in the Sri Lankan culture. When the person with dementia develops difficulties in performing executive functions, they find these routines difficult. This again was a new aspect we found in our study, which has not been reported in the literature elsewhere.

There are many modifications that can be done in the dining environment to reduce distractions. These include minimizing noises, maintaining contrasts between the table cloth, plate and food, avoiding patterned utensils and removing unnecessary objects from the table (16). Adaptive utensils also improve the functional state and independence in people with dementia (10). But none of 
the caregivers in our study described any similar modifications except use of non-fragile plates and cups. They appeared not to be aware or not to have considered these modifications. Lack of access to information on these aspects, disconnection from support groups and inadequate attention to these issues in clinical practice might have been contributory.

In some instances, caregivers appeared to take over responsibilities because it is easier in this way to finish a particular task. The cultural concept of performing everything on behalf of 'ill' individuals may also have contributed to this response. It has been shown that patients are happy to be independent in eating as long as possible (17). Respecting the dignity of the patient during mealtimes is very important. During this study it was noted that in some instances the individual is dishonoured in a subtle manner, in the process of being looked-after, even when patients are being looked after by family caregivers. This can affect the psychological well-being and functional status of individuals with mild to moderate dementia who have some degree of insight.

\section{Limitations}

The findings of this study are based on a single focus group discussion. Having several discussions with several groups would have provided more information. Considering the group dynamics and stigma on people with dementia there is a possibility of bias in the information gathered.

\section{Conclusions}

This study reveals multiple mealtime difficulties experienced by caregivers looking after persons with early dementia, which is especially applicable to the Asian culture, which has not been explored earlier. Use of this knowledge in routine clinical practice would improve the quality of care.

\section{Acknowledgments}

The authors wish to acknowledge all participants of the study and staff of the University Psychiatry Unit, National Hospital of Sri Lanka for their cooperation in the study.

\section{Declaration of interests}

None declared.

\section{Funding}

This research received no specific grant from any funding agency in the public, commercial, or not-for-profit sectors.
DGH Damayanthi, Postgraduate Institute of Medicine, University of Colombo, Sri Lanka

AMNL de Silva, Department of Clinical Sciences, Faculty of Medicine, General Sir John Kotelawala Defence University, Sri Lanka

CU Suraweera, IAGMP Gunathilake, University

Psychiatry Unit, National Hospital of Sri Lanka

Corresponding author: H Damayanthi

Email: hasitha.dg@gmail.com

http://orcid.org/0000-0002-7520-8282

\section{References}

1. Hugo J, Ganguli M. Dementia and cognitive impairment: epidemiology, diagnosis, and treatment. Clin geriatr med 2014; 30: 421-42.

2. Schulz R, Martire LM. Family caregiving of persons with dementia: prevalence, health effects, and support strategies. Am J Geriatr psychiatry 2004; 12: 240-9.

3. Prince M, Wimo A, Guerchet $\mathrm{M}$, et al. World Alzheimer Report 2015: the global impact of dementia: an analysis of prevalence, incidence, cost and trends. London; Alzheimer's Disease International; 2015.

4. Berkhout AM, Cools HJ, Van Houwelingen HC. The relationship between difficulties in feeding oneself and loss of weight in nursing-home patients with dementia. Age Ageing 1998; 27: 637-41.

5. Kai K, Hashimoto M, Amano K, Tanaka H, Fukuhara R, Ikeda M. Relationship between eating disturbance and dementia severity in patients with Alzheimer's disease. PLoS One 2015; 10(8).

6. Watson R. Undernutrition, weight loss and feeding difficulty in elderly patients with dementia: a nursing perspective. Rev Clin Gerontol 1997; 7: 317-26.

7. American Geriatrics Society Ethics Committee Clinical Practice Models of Care Committee. American Geriatrics Society feeding tubes in advanced dementia position statement. J Am Geriatr Soc 2014; 62: 1590-3.

8. DiBartolo MC. Careful hand feeding: a reasonable alternative to PEG tube placement in individuals with dementia. J Gerontol Nurs 2006; 32: 25-33.

9. Volicer L. Goals of care in advanced dementia: quality of life, dignity and comfort. J Nutr Health Aging 2007; 11: 481.

10. Murphy JL, Holmes J, Brooks C. Nutrition and dementia care: developing an evidence-based model for nutritional care in nursing homes. BMC Geriatri 2017; 17: 55.

11. Maguire M, Delahunt B. Doing a thematic analysis: A practical, step-by-step guide for learning and teaching 
scholars. The All Ireland Journal of Teaching and Learning in Higher Education. 2017; 9(3).

12. Albanese E, Taylor C, Siervo M, Stewart R, Prince MJ, Acosta D. Dementia severity and weight loss: A comparison across eight cohorts. The 10/66 study. Alzheimers Dement 2013; 9: 649-56.

13. Chang CC, Roberts BL. Feeding difficulty in older adults with dementia. J Clin Nurs 2008; 17: 2266-74.

14. Pasman HRW, The BAM, Onwuteaka-Philipsen BD, Van Der Wal G, Ribbe MW. Feeding nursing home patients with severe dementia: a qualitative study. J Adv Nurs 2003; 42: 304-11.

15. Wang PN, Yang CL, Lin KN, et al. Weight loss, nutritional status and physical activity in patients with Alzheimer's disease. J Neurol 2004; 251: 314-20.

16. Chang CC, Roberts BL. Strategies for feeding patients with dementia. AJN The Am J Nurs 2011; 111: 36-44.

17. Johansson L, Christensson L, Sidenvall B. Managing mealtime tasks: Told by persons with dementia. J Clin Nurs 2011; 20: 2552-62. 